\title{
Comparison of oncologic outcome between open versus laparoscopic distal pancreatectomy in patients with pancreatic ductal adenocarcinoma: Analysis with 1,202 patients in national database
}

\author{
Hongbeom KIM, Jae-Seung KANG, Heeju SON, Youngmin HAN, Wooil KWON, Jin-Young JANG*
}

Department of Surgery, Seoul National University Hospital, Seoul, Korea

Introduction: Although the lack of high-level evidence, laparoscopic distal pancreatectomy (LDP) in pancreatic ductal adenocarcinoma (PDAC) is being performed by many surgeons due to the development of surgical techniques. The purpose of this study was to investigate the long-term oncologic outcomes of LDP in PDAC through propensity score matching (PSM) analysis with large-scale national database.

Methods: Patients who received DP for PDAC registered in the official database of KAHBPS (KOTUS-BP) were enrolled. For longterm oncologic outcome analysis, 5-year overall survival rate (5YOSR) and 5-year disease-free survival rate (5YDFSR) were compared to open DP (ODP).

Results: From May 2001 to December 2016, 1,202 patients with detailed operative information and sufficient pathological data in 16 hospitals were selected. There were 846 ODP cases (70.4\%) and 356 LDP cases (29.6\%). In the ODP group, more aggressive surgery was performed, the pathologic stage was higher, R0 rate was higher $(81.2 \%$ vs. $77.0 \%, p<0.001)$ and there was a greater number of retrieved lymph nodes (15.3 vs. 13.5, $p=0.002)$ than the LDP group. PSM was performed with 8 variables including age, sex, operation period, combined organ resection, major vessel resection, $\mathrm{R}$ status, $\mathrm{T}$ stage, and $\mathrm{N}$ stage. After matching, the 5YOSR of ODP and LDP were $37.3 \%$ and $41.4 \%(p=0.150)$, and 5YDFSR was $23.4 \%$ and $27.2 \%(p=0.332)$, respectively. Prognostic factors for 5YOSR were R status, $\mathrm{T}$ stage, $\mathrm{N}$ stage, differentiation, and lymphovascular invasion.

Conclusions: LDP was performed in a selected group, and within this group, it had comparable long-term oncologic outcome to ODP in patients with PDAC. 